\title{
A remarkable double helix in the V838 Mon nebula
}

\author{
P. Carlqvist
}

\author{
Alfvén Laboratory, Royal Institute of Technology, 10044 Stockholm, Sweden \\ e-mail: per.carlqvist@alfvenlab.kth.se
}

Received 8 October 2004 / Accepted 22 February 2005

\begin{abstract}
In the beginning of 2002 the previously unnoted star V838 Mon had a powerful outburst. The star is surrounded by pre-existing, dusty clouds which are illuminated by the star in an expanding, parabolic layer. Spectacular images captured by the Hubble Space Telescope Advanced Camera for Surveys show that the clouds are to a great extent built up by filaments and concentric shells. One of the most remarkable features is a filamentary structure forming a double helix. The structure, which has a projected length and width of $\sim 99^{\prime \prime}$ and $\sim 1$ ". 4 , respectively, points almost radially towards V838 Mon. In order to reveal the geometry of the double helix in some more detail, a three-dimensional computer model of the structure has been constructed. The model also assists in determining the expansion rate of the light echoes along the double helix. By means of the expansion rate and the tilt of the double helix the distance to V838 Mon is determined to be $2.4 \pm 0.5 \mathrm{kpc}$. A theory of the double helix, based on a magnetized and twisted filament, is presented. Dynamic and magnetic forces play an essential role in the shaping of the double helix. The theory is supported by a mechanical analogy model. Double helices in other cosmic environments are also discussed.
\end{abstract}

Key words. stars: individual: V838 Mon - stars: winds, outflows - cicumstellar matter - ISM: clouds - plasmas ISM: magnetic fields

\section{Introduction}

In the first few months of 2002 the previously unnoted star V838 Monocerotis underwent a powerful outburst. The development of its brightness, mainly observed by ground-based instruments, was highly unusual (Munari et al. 2002, and references therein). The first signs of the outburst were noticed in early January, 2002 when the brightness rose some five magnitudes to $V \approx 10 \mathrm{mag}$. In the beginning of February the brightness increased again, and reached a prominent and well-defined peak of $V=6.75 \mathrm{mag}$ on February 6. Simultaneous peaks were observed also in the $B$ - and $I$-bands. After a quick decline two new and less well-defined brightness maxima took place on about March 8 and 28, now in the $V$ - and $I$-bands only. In late April the outburst was over. Throughout the outburst the colour of the star changed appreciably. During the first prominent maximum it was yellowish white. Later on it became more reddish while finishing as deeply red.

The star and its close environment were imaged by means of the Hubble Space Telescope Advanced Camera for Surveys (ACS) on April 30, May 20, September 2, October 28, and December 17, 2002 (Bond et al. 2003). The images show that the star is surrounded by a bright, nearly circularly limited nebula. The nebula is not self-emitting but contains dust that reflects light from the central star. The set of images reveals that the nebula appears to expand with superluminal speed. Munari et al. (2002) have interpreted the expansion in terms of light echoes of the star in the surrounding cloud. At any given time, the reflected light comes from a parabolically shaped layer. The illuminated layer successively moves outwards from the star giving an impression of a superluminal expansion.

Normally an image shows a physical object in a twodimensional projection. Hence, it is generally hard to grasp the depth of the object unless the shape of the object is known beforehand. As regards the cloud surrounding V838 Mon, however, we have a unique opportunity to get a glimpse of the third dimension. The reason for this is that the nebula is scanned by the outwardly propagating, parabolic layer. The images of April 30 and May 20 show that the nebula is composed of bright filaments, concentric rings or arcs, and a central cavity, probably created by previously ejected matter. On the image of May 20 a bright braid consisting of at least two filaments may be seen along the eastern rim of the cavity. To the west of the cavity there are three or four filaments, some of which are wavy. The more recent images reveal still more filamentary clouds, and some of these seem to be twisted. The filamentary structures, which are supposed to be aligned by magnetic fields, form various angles with the radial direction. Even arcs are present in the cloud.

\section{A double helix in the surrounding cloud}

One of the most intriguing structures in the nebula surrounding V838 Mon is seen $\sim 11^{\prime \prime}-17^{\prime \prime}$ north-west of the star (PA = $322^{\circ}$ ) on the image of September 2 (Fig. 1). An elongated structure, to some extent resembling a barber's pole, here 


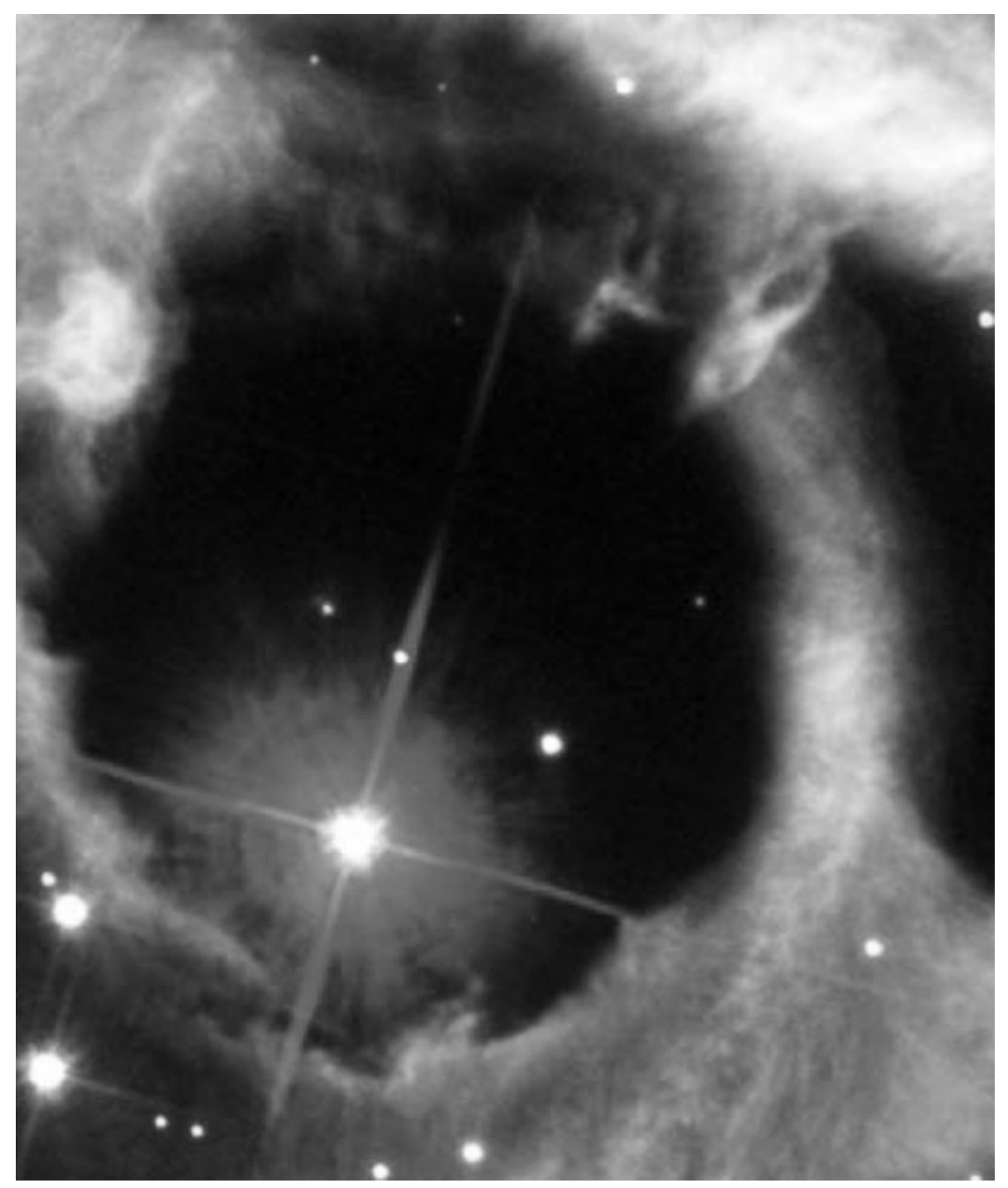

Fig. 1. V838 Mon and the double helix imaged by the Hubble Space Telescope ACS on September 2, 2002 (Credit: NASA, ESA and Bond (STScI) et al. 2003) The head of the double helix is situated $\sim 11^{\prime \prime}$ from V838 Mon in PA $=322^{\circ}$. Two serpentine filaments, coalescing in the head, appear to form the double helix. The symmetry axis of the double helix nearly coincides with the radial direction towards V838 Mon. The image covers $20^{\prime \prime} \times 24^{\prime \prime}$. North is up.

extends radially inwards, towards V838 Mon. The pole consists of two filaments which appear to be twisted around each other in the form of a double helix. An inspection of the image reveals that the symmetry axis of the double helix is almost precisely directed towards the central star. The deviation is less than two degrees. An enlargement of the area containing the double helix is shown in Fig. 2a. We denote the two helical filaments by Filament I and Filament II, where Filament I is above Filament II in the interval $\sim 11^{\prime \prime} .7-13^{\prime \prime} 3$ and below it in $\sim 13^{\prime \prime}$. $4-15^{\prime \prime}$. 7 . The inner part of the double helix, facing V838 Mon, is clearly reddish while the outer part is bluishwhite reflecting the evolution of the colour of V838 Mon during the outburst. A remarkable fact is that the outer part of Filament I appears to be more bluish than the outer part of Filament II thus showing a colour shift perpendicular to the radial direction, too.

The double helix can also be followed on the image of October 28 (Fig. 2b). The illuminated, parabolic layer has now moved some distance outwards and we cannot any more see the inner parts of the double helix. Instead, the image shows an intermediate section of the double helix with some overlap of the previous image. The image of December 17, reveals that the structure extends still further out from V838 Mon (Fig. 2c). The double helix structure is not as clear and evident in the outer parts as in the inner ones. At its outer end the double helix seems to split into two filaments which tend north-eastwards and north-north-westwards. In Fig. $2 d$ the three images are schematically combined into one picture. The total projected length of the double helix is about $9^{\prime \prime}$ while the width is $\sim 1$." 4 .

\section{The double-helix mechanism}

It is legitimate to ask how such a strange structure as the double helix may once have arisen in the clouds surrounding V838 Mon. Before trying to answer this question it is helpful to consider another kind of object, the twisted 

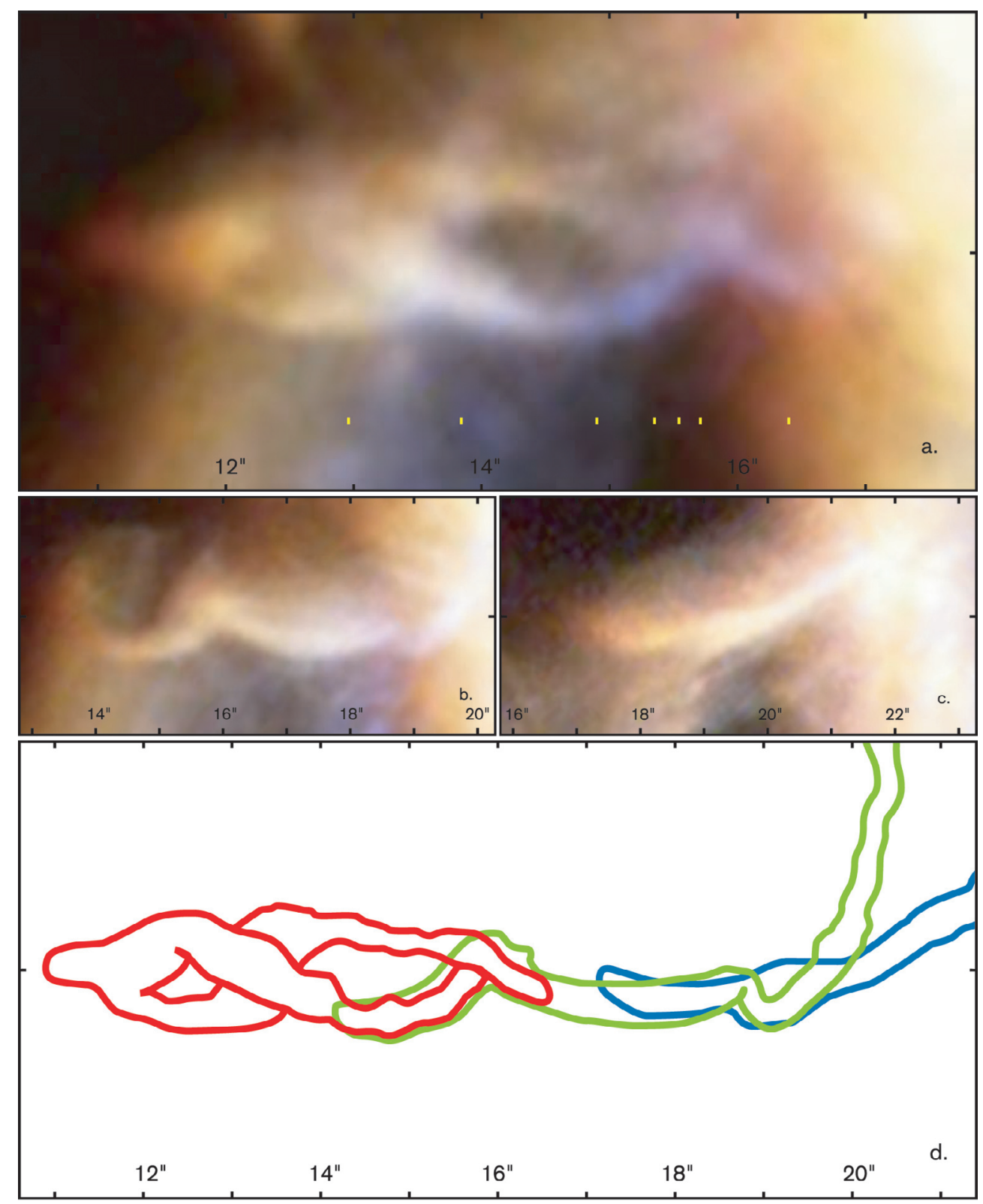

Fig. 2. ACS images of the double helix structure on a) September 2, 2002; b) October 28, 2002; and c) December 17, 2002 (Credit: NASA, ESA and Bond (STScI) et al. 2003). The frames are oriented so that the axis of the double helix, marked by tick marks to the left and to the right, is horizontal. The horizontal scales show the angular distance from V838 Mon along the axis. For the tick marks below the double helix in a), please refer to Fig. 5. A schematic amalgamation of the images of September 2 (red), October 28 (green), and December 17 (blue) is shown in d).

elephant trunks. The elephant trunks are dark, elongated, molecular clouds which are found mainly on the fringes of young H II regions. Generally, the trunks point radially inwards towards young, luminous stars in the central part of the $\mathrm{H}$ II region. Powerful winds and radiation from these stars cause the $\mathrm{H}$ II region to expand, and surrounding cloud matter to be swept along by an expanding shell. The elephant trunks mostly exhibit an internal filamentary structure which in many cases appear to be twisted (Carlqvist et al. 1998, 2002, the latter hereafter called CGK02). At their outer end the trunks often split into two or more filamentary legs which seem to connect to the shell.
Recently a new theory of such elephant trunks has been advanced (CGK02; Carlqvist et al. 2003, hereafter called CGK03). The theory assumes that a magnetized filament (a magnetic rope) is present in the molecular cloud surrounding the $\mathrm{H}$ II region. When the expanding shell hits the filament one out of two things may take place. If on the one hand the gas density in the filament is comparable to or smaller than the density of the ambient gas, the filament will simply be swept along by the shell and incorporated in it. If on the other hand the filament contains a local mass condensation, part of the filament may lag behind the shell due to its larger inertia. Although the filamentary gas is cold and contains only a relatively small 
fraction of ions and electrons it must still be considered a plasma with a large magnetic Reynold number $\left(R_{\mathrm{m}} \gg 1\right)$. This means that the magnetic field lines are well frozen in to the plasma. Hence, the filamentary matter and the magnetic field have to keep together as a unity. In the case the magnetic field lines inside the filament are straight or only moderately twisted, the filament will be stretched into the shape of a $V$ with the mass condensation situated near the apex of the $V$. The two upper parts of the $V$ connect to the shell containing the rest of the filament.

If, in contrast, the magnetic field lines are twisted beyond a certain critical limit, the filamentary parts near the apex of the $V$ will be twisted into a double helix so that the shape of the filament rather resembles a $Y$ (CGK03). The stem of the $Y$, containing the mass condensation, points towards the centre of the H II region. This part is identified with the elephant trunk.

The transformation of the filament into a double helix in the stem of the $Y$ may be considered a good example of selforganization. First, the filamentary system is forced to a state far from equilibrium. This is attained by a progressive turning of the filament, e.g., by convective processes, so that the internal magnetic field is increasingly twisted. When the system has been forced sufficiently far from equilibrium it reaches a critical point corresponding to the point of bifurcation. Beyond this point the system enters a completely new phase of development. Inside the filament the magnetic field is not further twisted. Instead, the whole filament is twisted into a double helix. The system has thereby been brought to a higher degree of organization.

The mechanism leading to the double helix has been studied both theoretically and by means of a mechanical analogy model (CGK02; CGK03). The analogy model consists of a bunch of elastic strings which is stretched between two fixed points of attachment. As indicated already by Faraday, elastic strings are similar to magnetic fields as regards the action of the forces. Half-way between the points of attachment the bunch is loaded by a weight simulating the inertia force of the mass condensation. Experiments performed with the analogy model show that if the bunch of strings is not twisted at all or only moderately twisted the weight will stretch the bunch into a simple $V$-shape. But if the twist goes beyond a certain critical limit, the part of the bunch being closest to the weight will be transformed into a double helix, so that the general shape of the bunch resembles a $Y$ (see Fig. 3 in CGK02). The reason for this is that the system naturally tends towards an energy minimum while maintaining the helicity of the magnetic field constant. Similar results have also been obtained with a corresponding theoretical model comprising a magnetized filament (CGK03). Here, too, numerical calculations show that the filament is transformed into a double helix as soon as the magnetic field lines are twisted beyond a certain critical limit.

The models can, however, be considered only first approximations. The reason for this is that they represent the steady state while the actual double helix mechanism is likely to be dynamic since it includes inertia forces. The degree of accuracy of the models depends on the ratio of the time scale for the change of the double helix and the time needed for an Alfvén wave to propagate across the system. If this ratio is much larger than

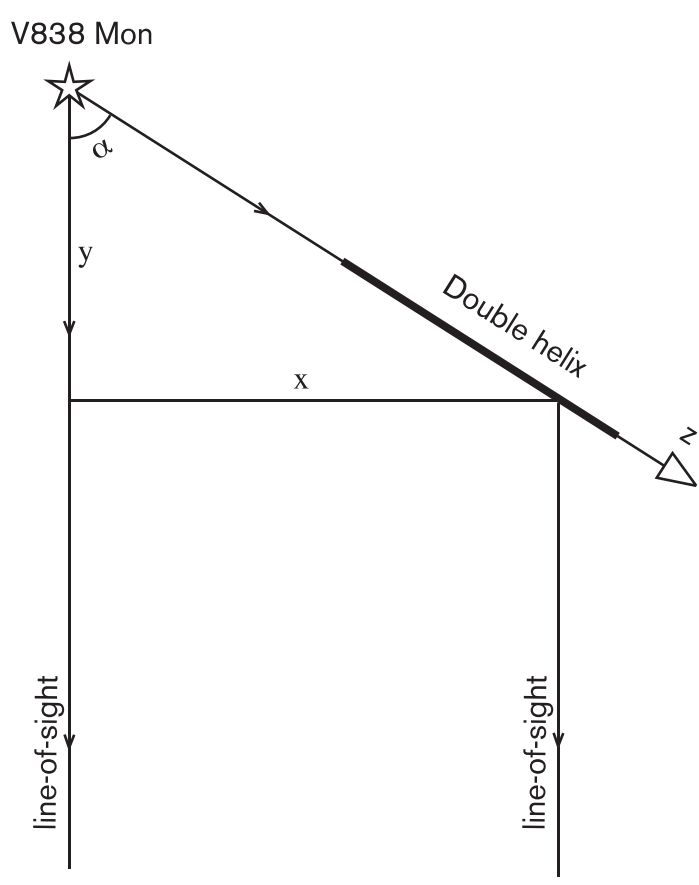

Fig. 3. Basic geometry of the V838 Mon - double helix system. The $z$-axis, which is taken to coincide with the axis of the double helix, has its origin in V838 Mon, and forms the angle $\alpha$ with the line-ofsight. The light from V838 Mon may either propagate directly to the observer or be echoed from the double helix.

unity, the time development may be regarded as a sequence of steady states implying a good accuracy of the models.

The double helix next to V838 Mon has several basic properties in common with the elephant trunks. Besides being elongated and twisted, both kinds of object point radially inwards towards activity centra. Moreover, the environments of the objects are fairly similar. Around V838 Mon are concentric shells indicating previous ejections of matter from the star just as there are expanding shells around the HII regions where the elephant trunks occur. Hence, it is natural to suggest that the double helix next to V838 Mon is produced by the same type of double-helix mechanism as the one being active in the elephant trunks.

\section{Basic geometry of the double helix - V838 Mon system}

As pointed out above, the image of September 2, 2002 (Fig. 1) shows that the symmetry axis of the double helix is very nearly directed towards V838 Mon. This alignment would be a remarkable coincidence if it were purely accidental, as it is in consonance with the double helix formation scenario discussed above. It is therefore reasonable to assume that the double helix is actually directed towards V838 Mon in all three dimensions. The resulting geometry of the double helix - V838 Mon system is schematically shown in Fig. 3. The z-axis, having its origin in V838 Mon and coinciding with the axis of the double helix, forms the angle $\alpha$ with the line-of-sight. With the basic geometry considered it is comparatively easy to interpret the 
expansion of the light echoes along the axis of the double helix. From the figure we find

$z-y=c \Delta t$

where $c$ is the speed of light and $\Delta t$ is the time lapse between a light signal arriving directly from V838 Mon and the corresponding light echo from the axis of the double helix. Using Eq. (1) we obtain the projected distance between V838 Mon and the point of reflection

$x=\frac{c \Delta t \sin \alpha}{1-\cos \alpha}$.

Since $\alpha$ is constant $x$ is proportional to $\Delta t$. This means that the light echo should be seen to propagate along the axis of the double helix with the constant speed

$v=\frac{x}{\Delta t}=\frac{c \sin \alpha}{1-\cos \alpha}$.

Equation (3) may also be expressed as

$\cos \alpha=\frac{1-f^{2}}{1+f^{2}}$,

where $f=c / v$. Substituting $v$ by the apparent expansion rate of the light echoes, $u$ (arcsec/day), we obtain the distance to V838 Mon (kpc)

$d=0.1731 \frac{1}{u f}$.

Thus, if we can determine $u$ and $\alpha$ we can also find the distance $d$.

\section{Estimate of the distance to V838 Mon}

The apparent expansion rate of the light echoes along the double helix may be found from the ACS-images in various ways. For instance, it can be estimated by dividing the apparent distance (arcsec) from V838 Mon to the inner red part of the double helix filament on the image of October 28, 2002 by the time that has lapsed since the end of the outburst. Using this procedure we obtain $u=0.076 \pm 0.004$ arcsec/day. The uncertainty is primarily due to the difficulty in identifying the exact time of release of the last red rays of light of the outburst, later reflected in the inner part of the filament.

If, instead, we use the interval between the images of September 2 and October 28 to calculate the expansion rate for the inner red part of the double helix, we find the different value $u \approx 0.042 \mathrm{arcsec} / \mathrm{day}$. This value is consistent with a declining red emission of V838 Mon already in the middle of December 2001, a result that obviously contradicts the observations. The resolution of this paradox is that the double helix of September 2, 2002 does not reach far enough inwards to be illuminated by the last red rays of the outburst of V838 Mon. This means that even the innermost part of the double helix can be seen on the image of September 2.

Using the expansion rate of the light echoes obtained above we have tried to identify the variations of the brightness and colour in the double helix with the observed variations of the intensity and colour of V838 Mon (Fig. 1 in Bond et al. 2003). It turns out, however, that such an identification is possible to make only in broad terms. The primary reason for this shortcoming is to be sought in the fact that the expansion rate of the light echoes along the axis of the double helix can only be considered a rough estimate. Since the filaments are supposed to spiral around the axis, the light echoes from them will sometimes arrive earlier and sometimes later as compared with the light echo from an imaginary, straight filament along the axis. Hence, the expansion rate of the echo must in reality be modulated.

To study this modulation we consider a very simple, threedimensional model of the double helix. As a basis of the model we choose the image of September 2, 2002 (Fig. 2a) which shows the double helix most clearly. The wavelength of each of the helical filaments is supposed to be constant while the radius of the helices varies as

$R=R_{0}\left[1-\mathrm{e}^{-2\left(z-z_{0}\right) / R_{0}}\right]$, for $z \geq z_{0}$.

This means that the radius is approximately constant in most of the double helix, but quickly tends to zero near the head at $z=z_{0}$, just as the image shows. From the image we find that $R_{0}=0$.'55. The two helices start from $z=z_{0}$, corresponding to $x=11^{\prime \prime} 6$, with individual phase angles which both can be varied in an appropriate way. We consider the projection of the model filaments on the celestial sphere, and adjust the projected wavelength $\lambda_{\text {proj }}$ and the phase $\phi_{0}$ at $z=z_{0}$ of each of the helical filaments so that the intersections of the filaments and the axis of the double helix agree with the corresponding intersections on the image ( $x=13^{\prime \prime} .3$ and $15^{\prime \prime} 7$ for Filament I and $x=13^{\prime \prime} 4$ and 15.' 7 for Filament II).

A glance at Fig. 2a indicates that the double helix is seen rather much sideways. In order to determine the tilt angle $\alpha$ more accurately we show in Fig. 4 the projection of the double helix model on the celestial sphere for a few different values of $\alpha$. As is clear from the figure, the projection agrees reasonably well with the image of the double helix for tilt angles in the interval $\sim 75^{\circ}-105^{\circ}$. The values of the projected wave length $\lambda_{\text {proj }}$ and phase $\phi_{0}$ for both of the helical filaments are also shown in Fig. 4. It is realized, that if the filaments are to form a fairly regular double helix, $\lambda_{\text {proj }}$ of the two filaments must not differ too much. From the variation of $\lambda_{\text {proj }}$ with $\alpha$ it is found that the wave lengths are equal for $\alpha=87^{\circ}$. It also turns out that the difference of the phases of the two helical filaments at $z=z_{0}$ is $180^{\circ}$ for this particular tilt angle, just as it should be for a smooth connection of the filaments. Since the double helix in Fig. 2a appears to be fairly regular, the wave lengths of the filaments are expected to differ by no more than 10-20\% from one another. A difference within $20 \%$ corresponds to tilt angles in the interval $75^{\circ}-99^{\circ}$.

Next step in the adaption process is to adjust the apparent expansion rate, $u$, along the $x$-axis so that some of the intensity and colour variations in both of the filaments can be identified with some of the more prominent features of the multicolour light curves of V838 Mon (e.g., Bond et al. 2003) echoed from the model filaments. This is a delicate task which is not quite straightforward - not even with the specially adopted model. One important reason for this is that there are natural density 
$\begin{array}{llll}\alpha=60^{\circ} & \text { Filament I : } & \lambda_{\text {proj }}=3 " .70 ; & \phi_{0}=76^{\circ} \\ & \text { Filament II : } & \lambda_{\text {poj }}=5 " .70 ; & \phi_{0}=356^{\circ}\end{array}$

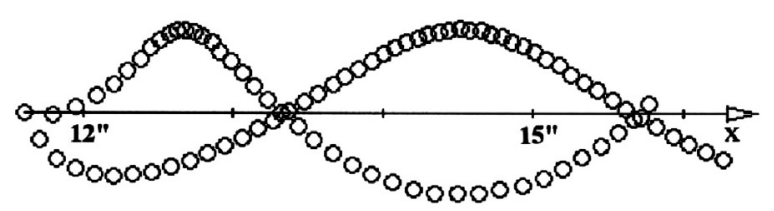

$\alpha=75^{\circ} \quad$ Filament I : $\quad \lambda_{\text {proj }}=4^{\prime \prime} .23 ; \quad \phi_{0}=114^{\circ}$

Filament II : $\quad \lambda_{\text {proj }}=5^{\prime \prime} .17 ; \quad \phi_{0}=335^{\circ}$

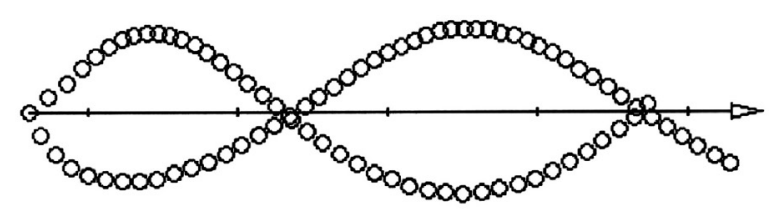

$\alpha=90^{\circ} \quad$ Filament I : $\quad \lambda_{\text {proj }}=4^{\prime \prime} .80 ; \quad \phi_{0}=143^{\circ}$

Filament II : $\quad \lambda_{\text {proj }}=4 " .60 ; \quad \phi_{0}=311^{\circ}$

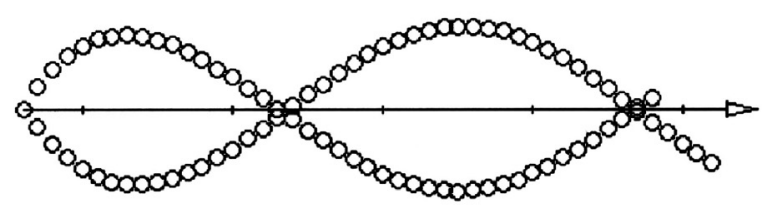

$\begin{array}{llll}\alpha=105^{\circ} & \text { Filament I : } & \lambda_{\text {proj }}=5^{\prime \prime} .37 ; & \phi_{0}=165^{\circ} \\ & \text { Filament II : } & \lambda_{\text {proj }}=4^{\prime \prime} .03 ; & \phi_{0}=278^{\circ}\end{array}$

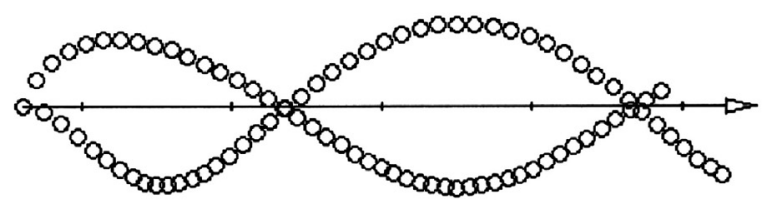

$\begin{array}{llll}\alpha=120^{\circ} & \text { Filament I : } & \lambda_{\text {proj }}=5^{\prime \prime} .90 ; & \phi_{0}=184^{\circ} \\ & \text { Filament II : } & \lambda_{\text {pro }}=3^{\prime \prime} .50 ; & \phi_{0}=237^{\circ}\end{array}$

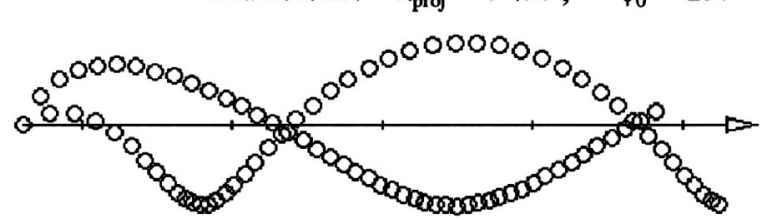

Fig. 4. A three-dimensional model of the double helix seen under various tilt angles $\alpha$. The intersections of the $x$-axis and the two helical filaments in the projection of the model have been brought to coincide with the corresponding intersections on the September 2 image of V838 Mon (Fig. 2a). The two filaments, which connect in the head of the double helix, are represented by a series of projected spheres the consecutive centra of which are separated by a constant amount in the $z$-direction. The projected wave length $\lambda_{\text {proj }}$ and the phase $\phi_{0}$ at $z=z_{0}$ are also given for both of the filaments.

fluctuations in the filaments which give rise to intensity variations that are not associated with variations of the light curves. Most suitable to identify is the echo of the intensity minimum in the near-infrared $I$ band about the heliocentric Julian date (HJD) 2452320 (February 14, 2002). This minimum also includes a distinct change of colour that is comparatively easy to recognize. Once an apparent expansion rate is established, it becomes possible to identify other features of the light curves in the images of the double helix (Figs. 5 and 2a). It is found that only right-handed helices can be adjusted properly. This finding agrees well with the impression one gets from the image of the double helix in Fig. 2a. In Figs. 5 and 2a the matching has been done for $\alpha=87^{\circ}$ but model calculations also offer good matches for other tilt angles in the fairly broad interval $45^{\circ}-135^{\circ}$ where the expansion rate stays remarkably constant. The apparent expansion rate found is $u=0.0758 \pm 0.0008$ arc$\mathrm{sec} / \mathrm{day}$. This value closely agrees with the value derived above by means of a different method. The uncertainty reflects the errors in the measuring of the features on the image and light curves.

Figure 5 also enables us to see why the right-hand part of Filament I appears bluer than the right-hand part of Filament II in Fig. 2a (cf. Sect. 2). Here, Filament I reflects light from an earlier and, hence, bluer epoch of V838 Mon while Filament II reflects light from a later and redder epoch. The apparently odd colour distribution found may thus be considered a proof of the three-dimensional nature of the double helix.

With the expansion rate and tilt angle derived we are now prepared to determine the distance to V838 Mon. Figure 6 shows the distance $d$ as a function of the tilt angle $\alpha$ for $u=0.0758 \mathrm{arcsec} / \mathrm{day}$ according to Eq. (5). With a tilt angle of $\alpha=87^{\circ} \pm 12^{\circ}$ we find the distance to V838 Mon to be $d=2.4 \pm 0.5 \mathrm{kpc}$. The uncertainty in $d$ mainly derives from the uncertainty in $\alpha$.

Above we have assumed that the axis of the double helix is directed towards V838 Mon in all three dimensions. However, we can not completely exclude the possibility that the axis may pass either in front of the star or behind it. A likely reason for such a configuration could be that the circumstellar material is distributed in an oblong geometry with the long axis directed towards the observer, or in some other non-spherical geometry. Hence, it may be of some interest to see what such a non-radial alignment for the double helix implies. For this purpose we have modified the model considered above so that V838 Mon is displaced a distance $s$, either behind the axis of the double helix $(s>0)$ or in front of it $(s<0)$, as measured along the line-of-sight. Using the same tilt angle of the double helix axis, $\alpha=87^{\circ}$, as before and identifying the intensity minimum at HJD 2452320 as shown in Figs. 2a and 5 we find that the expansion rate of the light echo along the axis of the double helix decreases asymptotically outwards towards a constant value which depends on $s$. The distance $d$ to V838 Mon is found to be larger than $2.4 \mathrm{kpc}$ if $s>0$ and smaller if $s<0$. In particular the distances $d=2.9 \mathrm{kpc}$ and $d=1.9 \mathrm{kpc}$, deviating from $2.4 \mathrm{kpc}$ by an amount that is equal to the error $\pm 0.5 \mathrm{kpc}$ derived above, are found for $s \approx \pm 1.5 \times 10^{-5} d$ (corresponding to $\sim 3$.'1). From the image of September 2 (Fig. 1), V838 Mon is found to be situated at an angular distance of not more than $0 ! 3$ from the axis of the double helix. With the above values of $s$ we find that the probability of finding V838 Mon closer to the double helix axis than $0 ! 3$ is less than one part in sixteen if the star is observed from an arbitrary direction. Hence, we may conclude that the initial assumption of $s=0$ appears to be very reasonable and most likely represents a good approximation. 


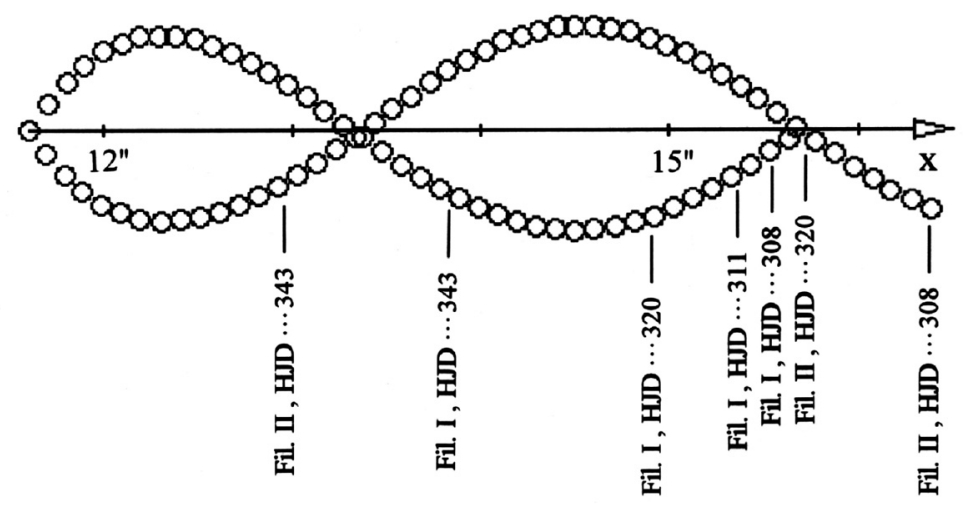

Fig. 5. The same type of projection of the three-dimensional model as shown in Fig. 4, but now for the preferred tilt angle $\alpha=87^{\circ}$. A few of the more prominent features of the multicolour light curves (Bond et al. 2003) echoed from the model filaments, have been adapted to the intensity and colour variations of the filaments in Fig. 2a. Thus HJD 2452320 represents a minimum in the $I$-band and a distinct change of colour. Further, HJD 2452311 indicates the bluish-white maximum of a prominent and narrow peak while HJD 2452308 shows the beginning of the peak. Another maximum, which is redder but less distinct, is found on about HJD 2452343 . Tick marks in Fig. 2a indicate the same dates and positions.

It may be of some interest to compare the distance derived with previous estimates. Using the apparent expansion rate of the light echo and an assumption of a spherically symmetric, circumstellar dust distribution Munari et al. (2002) derive a distance of $790 \pm 30 \mathrm{pc}$. Goranskii et al. (2002) find a somewhat larger distance, $1190 \pm 70 \mathrm{pc}$, based on the radius of the nebula in April 2002 and the expansion rate. In a more detailed consideration of the expansion rate of the light echoes and assuming spherical geometry of the circumstellar clouds, Bond et al. (2003) infer a distance to V838 Mon of $>2 \mathrm{kpc}$. Furthermore, from one polarization measurement they also obtain a lower limit of the distance of $\sim 6 \mathrm{kpc}$. By means of the expansion rate Crause (2003) finds a distance of $2.5 \mathrm{kpc}$ while Tylenda (2004) estimates the distance to $5 \mathrm{kpc}$ and $8.0 \pm 2.0 \mathrm{kpc}$ in two different regions of the surrounding cloud. Hence, it may be concluded that there is a considerable scattering of the distances derived.

From the tilt angle and distance derived we may draw some conclusions on the properties of the double helix. Using $d=$ $2.4 \mathrm{kpc}$ and $\alpha=87^{\circ}$ we find that the real length and width of the double helix is $\sim 3.2 \times 10^{15} \mathrm{~m}=2.2 \times 10^{4}$ ua and $\sim 5.0 \times 10^{14} \mathrm{~m}=$ $3.4 \times 10^{3}$ ua, respectively. The thickness of the filaments, which varies in the interval $0{ }^{\prime} 3-00^{\prime} 6$, corresponds to $1.1 \times 10^{14}-2.2 \times$ $10^{14} \mathrm{~m}=7 \times 10^{2}-1.4 \times 10^{3}$ ua. Furthermore, the wavelength of the helices in the most distinct part of the structure (Fig. 2a) is $1.7 \times 10^{15} \mathrm{~m}$. With $R_{0}=0$ ' 55 this corresponds to a pitch angle of the helices of $\sim 54^{\circ}$.

A peculiarity of the clouds surrounding V838 Mon is that they seem to contain only one distinct pole pointing towards V838 Mon. This contrasts with the HII regions which often house a number of radially directed elephant trunks. There may, however, be one more example of a radially directed, pole-like structure in the clouds near V838 Mon. This structure, which is seen about $3^{\prime \prime}$ north-east of the double helix (Fig. 1), is not as regular and clear as the double helix considered above. But it roughly points towards V838 Mon and seems to have two legs. Possibly it may be a less massive double helix that is presently dissolving because of winds and erosion.

\section{Double helices in other cosmic environments}

What may be learned from the double helix next to V838 Mon? Besides being a fascinating object in itself it may also offer important clues to the understanding of the general properties of double helices and, in addition, where to find other double helices in different environments. Previously we have mentioned that the twisted elephant trunks appear to be made up of dark filaments forming double-helix structures. Double helices are, however, likely to occur also in other cosmic sites.

For instance, it has recently been suggested that double helices are present in the Crab Nebula in the form of the so called "fingers" (Carlqvist 2004). The nebula contains a hot synchrotron plasma which expands outwards, and sweeps along an external, cooler gas. In the intermediate shock front there is a network of filaments. Many of the filaments have out-growths in the form of fingers which point radially inwards, towards the central part of the nebula. The fingers are mostly not structureless but consist of filaments. Some of the filaments show clear signs of being twisted. In the tips of the fingers, facing the centre of the nebula, there is generally a mass condensation.

Another site where a double helix is likely to exist is in the North Celestial Pole (NCP) Loop (Gahm 2003; CGK03). The NCP Loop is a huge (diameter $\sim 30^{\circ}$ ), annular formation of filamentary, molecular gas which stands out particularly well on IRAS images in $100 \mu \mathrm{m}$ (Heiles 1984). The formation constitutes the observable result of an oblong, expanding bubble which interacts with the surrounding gas (Meyerdierks et al. 1991). From the rim of the NCP Loop an elongated cloud, MBM 27-30 (Magnani et al. 1985; Pound \& Goodman 1997), hangs down towards the centre of the loop. The cloud has the dimensions $\sim 5^{\circ} \times 1^{\circ}$ and consists of several separate filaments which wind around one another in a helix-like geometry.

The examples given above indicate that there are several hallmarks associated with the double helices. Examples of such hallmarks are: i) a bubble being blown up by an enhanced internal pressure; ii) a shock front in the shell of the bubble; 
iii) a network of filaments in the shell; iv) an elongated structure pointing towards the centre of the bubble; v) twisted filaments in the elongated structure; vi) a mass condensation in the head of the structure; and often vii) two filamentary legs connecting the elongated structure with the shell.

The hallmarks may be useful in the search for other cosmic double helices. For instance it is tempting to consider the elongated, giant molecular cloud Orion A a double helix on these grounds. The cloud, having a length of $\sim 5^{\circ}$ and a thickness of $\sim 1^{\circ}$, mainly consists of a number of filaments which appear to spiral around the axis of the cloud (Bally et al. 1987; Bally 1989; Uchida et al. 1991; Nagahama et al. 1998). The cloud is compressed in its north-western part. In the south-eastern end there is an unusual structure that Nagahama et al. compare to a fish-tail. This structure partly encircles the hot and luminous star Saiph ( $\kappa$ Orionis) in the south-eastern corner of the constellation of Orion. Nagahama et al. discuss whether stellar winds from Saiph could possibly have shaped the "fish-tail", but find that this cannot be the case. A more likely interpretation, in our view, is that the fish-tail structure represents the filamentary legs connecting the Orion A cloud with a shell. But where is the shell to be found? If we look more closely to the region where the fish-tail structure ends we find that there is actually a very prominent shell present exactly at that position. The shell is marked by Barnard's Loop, an arc or incomplete ring with a diameter of $\sim 12^{\circ}$. The arc appears to touch Saiph and the fishtail. Barnard's Loop, which seems to be situated at about the same distance as the Orion A cloud, marks the eastern edge of a superbubble. Also Bally et al. favour the view that the Orion A cloud may have been caused by the expansion of the superbubble but their mechanism differs on essential points from the mechanism discussed here.

\section{Conclusions}

As a result of the powerful outburst of V838 Mon in 2002 clouds surrounding the star have successively been lit up. Light from the star is reflected by dusty gas in an expanding parabolic layer. Magnificent ACS images have revealed delicate details in the surrounding clouds. The images show that the clouds partly consist of concentric shells, probably created by previously ejected matter. The shells, in turn, seem to be interspersed with wavy and twisted filaments.

We draw attention to an elongated and twisted structure in the north-western part of the cloud which, in contrast to most of the filaments, stretches mainly in the radial direction. The elongated structure, which is present on the ACS images of September 2, October 28, and December 17, 2002, appears to consist of two filaments that are twisted around each another in the form of a double helix. The double helix has a radial extension of $\sim 9^{\prime \prime}$ with its innermost tip situated about $11^{\prime \prime}$ from V838 Mon. The thickness is $\sim 1$ ". 4 . From the image of September 2 it is clear that the axis of symmetry of the double helix deviates less than a few degrees from the radial direction. The inner parts of the double helix, facing V838 Mon, are deeply red while the outer parts are bluish white, reflecting the progressive change of colour of V838 Mon.

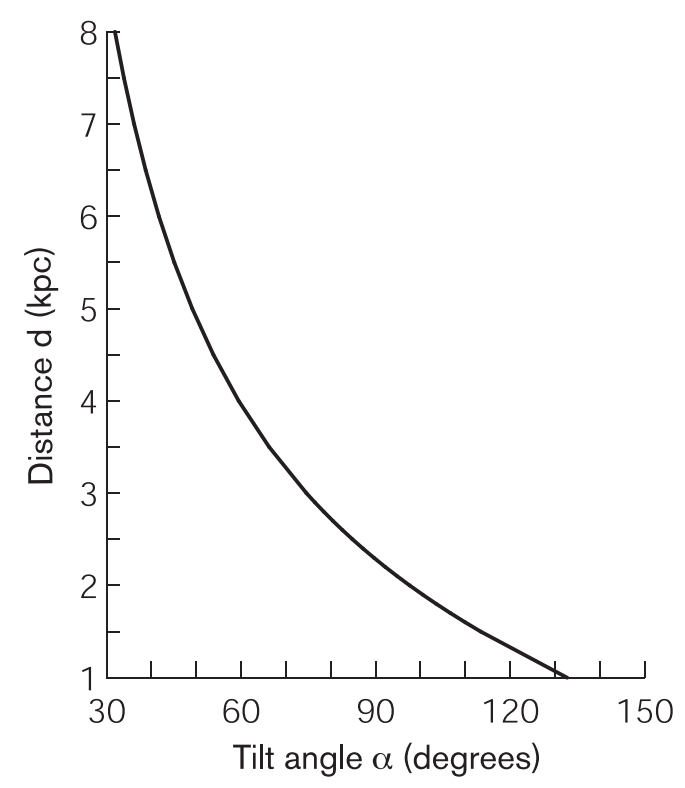

Fig. 6. Distance $d$ of the double helix and V838 Mon shown as a function of the angle $\alpha$ between the line-of-sight and the axis of the double helix for the apparent expansion rate of the light echo along the axis of the double helix $u=0.0758 \mathrm{arcsec} / \mathrm{day}$. The uncertainty of $u$ is contained within the thickness of the curve in the interval of interest.

The procedure for studying the three-dimensional structure of the double helix consists in adapting a three-dimensional model of the double helix so that its projection on the celestial sphere agrees as well as possible with the ACS images of the double helix. In addition to this, the model permits a calculation of how the light echoes of various phases of the outburst of V838 Mon proceeds along the projected filaments of the double helix. The colour and brightness variations observed in V838 Mon and reflected in the model filaments are then adjusted to the colour and intensity variations seen in the filaments imaged. As a result of the adjustment it has been found that i) the double helix is right handed; ii) the axis of the double helix forms the angle $87^{\circ} \pm 12^{\circ}$ with the line-of-sight; and iii) the distance to the double helix, and hence to V838 Mon, is $2.4 \pm 0.5 \mathrm{kpc}$. If the distance derived is correct, V838 Mon should be situated in or near the Perseus arm.

By means of the model calculations we also find that the double helix should not have been illuminated by the main outburst of V838 Mon and, hence, not visible before the end of June 2002 and after the end of January 2003. This is in agreement with images captured on e.g. May 20, 2002 (Bond et al. 2003) and October 21, 2003 (Henden 2003) on which no traces of the double helix can be seen. It would of course be of great value if the limits observed could be further tightened.

It may seem remarkable that such a complex structure as a double helix can be formed out of the clouds surrounding an outbursting star. We have suggested a mechanism which leads to such a structure under conditions that are likely to prevail in the clouds. The mechanism is similar to a mechanism active in a recently suggested theory of elephant trunks. This is not very surprising since the double helix next to V838 Mon has several properties in common with the elephant trunks. The mechanism presented comprises a magnetized filament, situated in 
the clouds surrounding V838 Mon. Owing to stellar winds or previous outbursts of V838 Mon, a bubble is supposed to expand around the star. The shell of the bubble sweeps along external cloud matter including at least part of the magnetized filament. In the case there is a mass condensation in the filament it will lag behind the shell because of its enhanced inertia. As a result the filament will be stretched into a $V$-shape. If the twisting of the magnetic field lines inside the filament is sufficiently large, the filament will also be twisted into a double helix next to the apex of the $V$ so that the general shape of the filament resembles a $Y$. The stem of the $Y$, which is identified with the double helix, points towards the central star. From an energetic point of view the double helix is driven by winds and ejections of matter but it is shaped by the combined action of inertia and magnetic forces. Both theoretical calculations and a mechanical analogy model lend support to the theory.

Double helices similar to the one close to V838 Mon are likely to exist also in many other cosmic environments. One example already mentioned is constituted by the twisted elephant trunks in young H II regions. Double helices have also been suggested to occur in supernova remnants like the Crab Nebula where they are supposed to form the fingers. Furthermore, in the NCP Loop at higher galactic latitudes there is a doublehelix-like structure, MBM 27-30, which stretches radially inwards from the loop. Another possible double helix is the giant molecular cloud Orion A. All these different environments where double helices are supposed to appear have a few hallmarks in common. Examples of such hallmarks are i) a bubble blown by an enhanced, internal pressure; ii) a shock front in the shell of the bubble; and iii) a network of filaments in the shell. Environments fulfilling these conditions are known to exist in many different cosmic sites. From this we may conclude that the double helix structure is probably not a rare phenomenon in cosmic plasmas.
Acknowledgements. I am indebted to G. Gahm for many valuable comments. My gratitude also goes to A. Brink and K. Carlqvist for kind assistance with the figures and the text, respectively. The project was supported by Carl Tryggers Foundation.

\section{References}

Bally, J. 1989, in Low Mass Star Formation and Pre-Main Sequence Objects, ed. B. Reipurth (Garching: ESO), 1

Bally, J., Langer, W. D., Stark, A. A., et al. 1987, ApJ, 312, L45

Bond, H. E., Henden, A., Levay, Z. G., et al. 2003, Nature, 422, 405

Carlqvist, P. 2004, Ap\&SS, 289, 47

Carlqvist, P., Gahm, G. F., \& Kristen, H. 2002, Ap\&SS, 280, 405 (CGK02)

Carlqvist, P., Gahm, G. F., \& Kristen, H. 2003, A\&A, 403, 399 (CGK03)

Carlqvist, P., Kristen, H., \& Gahm, G. F. 1998, A\&A, 332, L5

Crause, L. 2003, in Stars as Suns: Activity, Evolution and Planets, IAU Symp., 219, Sidney, Australia

Gahm, G. F. 2003, in Recent Res. Devel. Astrophys., Research Signpost, 1, 117

Goranskii, V. P., Kusakin, A. V., Metlova, N. V., et al. 2002, Astro. Lett., 28, 691

Heiles, C. 1984, ApJS, 55, 585

Henden, A. 2003, APOD,

http: //antwrp.gsfc.nasa.gov/apod/ap031205.html

Magnani, L., Blitz, L., \& Mundy, L. 1985, ApJ, 295, 402

Meyerdierks, H., Heithausen, A., \& Reif, K. 1991, A\&A, 245, 247

Munari, U., Henden, A., Kiyota, S., et al. 2002, A\&A, 389, L51

Nagahama, T., Mizuno, A., Ogawa, H., et al. 1998, AJ, 116, 336

Pound, M. W., \& Goodman, A. A. 1997, ApJ, 482, 334

Tylenda, R. 2004, A\&A, 414, 223

Uchida, Y., Fukui, Y., Minoshima, A., et al. 1991, Nature, 349, 140 